\title{
POSITIVE SOLUTIONS OF SYSTEMS OF SEMILINEAR ELLIPTIC EQUATIONS: THE PENDULUM METHOD
}

\author{
JOSEPH GLOVER
}

\begin{abstract}
Conditions are formulated which guarantee the existence of positive solutions for systems of the form

$$
\begin{aligned}
& -\Delta u_{1}+f_{1}\left(u_{1}, \ldots, u_{n}\right)=\mu_{1}, \\
& -\Delta u_{2}+f_{2}\left(u_{1}, \ldots, u_{n}\right)=\mu_{2} \text {, } \\
& \begin{array}{ccc}
\Delta & \vdots & \vdots
\end{array} \\
& -\Delta u_{n}+f_{n}\left(u_{1}, \ldots, u_{n}\right)=\mu_{n},
\end{aligned}
$$

where $\Delta$ is the Laplacian (with Dirichlet boundary conditions) on an open domain in $\mathbf{R}^{d}$, and where each $\mu_{i}$ is a positive measure. The main tools used are probabilistic potential theory, Markov processes, and an iterative scheme which is not a generalization of the one used for quasimonotone systems. Quasimonotonicity is not assumed and new results are obtained even for the case where $\partial f_{k} / \partial x_{j}>0$ for every $k$ and $j$.
\end{abstract}

0. Introduction. In this article, we introduce conditions which are sufficient to guarantee existence of a solution for a system of semilinear equations of the form

$$
\begin{array}{cc}
-\Delta u_{1}+f_{1}\left(u_{1}, \ldots, u_{n}\right) & =\mu_{1}, \\
-\Delta u_{2}+f_{2}\left(u_{1}, \ldots, u_{n}\right) & =\mu_{2}, \\
\vdots & \vdots \\
-\Delta u_{n}+f_{n}\left(u_{1}, \ldots, u_{n}\right) & =\mu_{n}
\end{array}
$$

on an open (bounded or unbounded) domain $E$ in $\mathbf{R}^{d}$, with Dirichlet boundary conditions. We assume each measure $\mu_{i}$ is positive, and we show that the solution is positive; i.e. $u_{i} \geq 0$ for every $i$. In fact, we give a constructive procedure for solving (0.1) with general elliptic operators in place of the Laplacian, and our methods even allow us to replace the Laplacian with certain integrodifferential operators. See $\S 4$ for other extensions. In $(0.1)$, the $\left(\mu_{k}\right)$ are assumed to be positive measures, and each $f_{k}: \mathbf{R}^{n} \rightarrow \mathbf{R}$ is continuous. The functions $f_{k}$ need not satisfy any special conditions such as $\partial f_{k} / \partial x_{j}>0$, but our methods yield new results even in that case. Previous approaches to solving (0.1) in a constructive way seem to have relied mainly on assuming that $(0.1)$ is a quasimonotone system and on using the method of sub- and supersolutions. For discussions of this approach, see [10 and 11]. Since we do not assume the system is quasimonotone, such methods do not apply, and even if the system is assumed to be quasimonotone, it is not clear how to obtain

Received by the editors April 3, 1986.

1980 Mathematics Subject Classification (1985 Revision). Primary 35J60; Secondary 31B05, 60J45.

Research supported by AFOSR Grant 85-0330 and NSF Grant DMS-8318204. 
upper and lower solutions in general. We introduce an iterative scheme in which the iterates do not increase or decrease monotonically, but instead oscillate. That is, we produce a sequence $\left(u_{k}\right)$ so that $u_{2} \leq u_{4} \leq \cdots \leq u_{2 k} \leq u_{2 k+1} \leq \cdots \leq u_{3} \leq u_{1}$. One might call this a pendulum method, since the iterates swing back and forth over the solution. Much more is known about solving one equation of the form $-\Delta u+f(u)=\mu$ than is known about solving (0.1). In his survey article [8], Lions discusses the single equation $-\Delta u+f(u)=\mu$, and he points out that it is important for applications to extend the results for the single equation to systems. See also $[\mathbf{1}, \mathbf{4}$, and $\mathbf{7}]$ for the case of one equation.

For general elliptic operators, positive measures, and arbitrary functions $f_{k}$, our hypotheses and results are a bit complicated to state, and we refer the reader to $\S \S 1$, 2, and 3. (In particular, Theorems (2.9) and (3.1) contain the main results.) In this introduction, we content ourselves with describing the hypotheses, methods, and results in the case where we keep the Laplacian with Dirichlet boundary conditions on a connected domain $E$ in $(0.1), f_{k}(0, \ldots, 0)=0$, and where

$$
\mu_{k}(d x)=g_{k}(x) d x \quad \text { with } g_{k}>0 \text { on } E,
$$

and for every $k$ and $j$, there is a constant $\rho_{k j}>0$ so that

$$
\partial f_{k} / \partial x_{j}\left(u_{1}, u_{2}, \ldots, u_{n}\right) \geq \rho_{k j} \quad \text { for } u_{1} \geq 0, \ldots, u_{n} \geq 0 .
$$

We emphasize that assumptions (0.2) and (0.3) are for the purposes of discussion in this section only: in $\S \S 2$ and 3 , we treat a much more general situation. Hypothesis (0.3) is a type of quasimonotonicity assumption. But even in this special case, one would need upper and lower solutions in order to apply the monotone iteration schemes of quasimonotone systems. It is not at all clear how to obtain upper and lower solutions, in general, and we completely avoid this problem. With hypotheses $(0.2)$ and $(0.3)$ in force, we make two crucial assumptions. Here is the first one. Let $V=(-\Delta)^{-1}$ on $E$, and let $M$ be Lebesgue measure on $E$. Assume

(i) $V g_{k}<\infty$ a.e. $(M)$ for every $k$.

(ii) For some $k, M\left(V g_{k}>V f_{k}\left(V g_{1}, \ldots, V g_{n}\right)\right)>0$.

Part (i) seems quite a reasonable assumption. From the potential theory point of view, part (ii) is interesting. Part (ii) is equivalent to saying that the measure $g_{k} d M$ is not a balayage of the measure $f_{k}\left(V g_{1}, \ldots, V g_{n}\right) d M$. This is quite often verifiable. For example, when we pass to discussing general measures $\mu$ in $\S 2$, the analogue of this requirement will be that " $\mu$ is not a balayage of the measure $G(V \mu) d M$ " (see $(2.4)$ ). It is interesting to note that this is automatically guaranteed if the dimension of the space $d$ is greater than or equal to 2 , and if the measure $\mu$ charges a point. Condition (ii) also implies $M\left(V f_{k}\left(V g_{1}, \ldots, V g_{n}\right)<\infty\right)>0$, and it is well known that we then have $V f_{k}\left(V g_{1}, \ldots, V g_{n}\right)<\infty$ a.e. $(M)$.

So if this first crucial assumption (0.4) holds, do the following. Set

$$
\begin{aligned}
J_{k}\left(u_{1}, u_{2}, \ldots, u_{n}\right) & =f_{k}\left(u_{1}, u_{2}, \ldots u_{n}\right) \quad \text { if } u_{1} \geq 0, \ldots, u_{n} \geq 0 \\
& =0 \quad \text { otherwise. }
\end{aligned}
$$

Set $u_{k}^{1}=V\left[g_{k}\right]$, and define inductively

$$
u_{k}^{j+1}=V\left[g_{k}-J_{k}\left(u_{1}^{j}, \ldots, u_{n}^{j}\right)\right]
$$


Then it is not difficult to see that $u_{k}^{2} \leq u_{k}^{4} \leq \cdots \leq u_{k}^{2 j} \leq u_{k}^{2 j+1} \leq \cdots \leq u_{k}^{3} \leq u_{k}^{1}$, so $u_{k}^{2 j}$ increases to a function $a_{k}$ and $u_{k}^{2 j+1}$ decreases to a function $b_{k}$. It is easy to show that $a_{k}=V\left[g_{k}-J_{k}\left(b_{1}, \ldots, b_{n}\right)\right]$ and $b_{k}=V\left[g_{k}-J_{k}\left(a_{1}, \ldots, a_{n}\right)\right]$. In addition, for each $k$, either $a_{k}<b_{k}$ on all of $E$, or $a_{k}=b_{k}$ on all of $E$. It is easy to show that $b_{k} \geq 0$. What is a bit more delicate is the fact that $b_{k}>0$ on $E$. To prove this, we use $g_{k}>0$ and Brownian motion (or, in general, the Markov process associated with the elliptic or integrodifferential operator). If $a_{k}=b_{k}$ for every $k$, then $b_{k}=V\left[g_{k}-J_{k}\left(b_{1}, \ldots, b_{n}\right)\right]$. Since $b_{k} \geq 0$, we may rewrite this as $b_{k}=V\left[g_{k}-f_{k}\left(b_{1}, \ldots, b_{n}\right)\right]$, and we are done (take the Laplacian of both sides-in the sense of distributions).

If $a_{k}<b_{k}$ for some $k$, we must restart the iteration. Now we need our second crucial hypothesis. Assume for each $k$ with $a_{k}<b_{k}$ :

There is a nonnegative function $F_{k}$ on $E$ and numbers $\delta_{k}$ and $\pi_{k}$ so that

(i) $\left\{F_{k}>0\right\}$ is contained in a compact set $K_{k}$ which is contained

$$
\begin{aligned}
\text { in } & \left\{a_{k}>\delta_{k}\right\} \cap\left\{b_{k}<\pi_{k}\right\} \text {. } \\
& \text { (ii) } F_{k} \leq J_{k}\left(b_{1}, b_{2}, \ldots, b_{k-1}, b_{k}-V F_{k}, b_{k+1}, \ldots, b_{n}\right)-J_{k}\left(a_{1}, \ldots, a_{n}\right) \text {. } \\
& \text { (iii) } F_{k} \leq \rho_{k k}^{2} V V\left(F_{k} 1_{\left\{a_{k}>V F_{k}\right\}}\right) . \\
& \text { (iv) } M\left(F_{k}>0\right)>0 .
\end{aligned}
$$

We show in $\S 2$ that the collection of all functions $F_{k}$ satisfying (i), (ii), and (iv) is nonempty: it contains lots of functions. One need only check whether or not one of them also satisfies (iii). Part (iii) should be thought of as a geometric condition: the larger the domain is, the more likely it is that (iii) will be satisfied. (For example, it often suffices to use an eigenfunction of $-\Delta$ for $F_{k}$. Let $\delta_{k}>0$ be small and $\pi_{k}>0$ be large. In "nice" situations, we expect $a_{k}$ and $b_{k}$ to be continuous functions, so $G_{k}=\left\{a_{k}>\delta_{k}, b_{k}<\pi_{k}\right\}$ will be open. Choose an open set $H$ so that the closure of $H$ is contained in $G_{k}$, and let $\phi$ be the first eigenfunction of $-\Delta$ on $H$ (with Dirichlet boundary conditions) and eigenvalue $\lambda>0$. Normalize $\phi$ so that $\sup \{\phi(x): x \in H\}=1$. Let $W$ be the inverse of $-\Delta$ on $H$-so $W \leq V$. Assume $V \phi$ is bounded by a constant $m$, and set $F_{k}=c \phi$ for some constant $c$ to be determined below. Then $\rho_{k k}^{2} V V\left(c \phi 1_{\left\{a_{k}>V c \phi\right\}}\right)=\rho_{k k}^{2} V V(c \phi)$ provided we choose $c$ so small that $\delta_{k} / c>m$. But $\rho_{k k}^{2} V V(c \phi) \geq \rho_{k k}^{2} c \phi / \lambda^{2}$. This is larger than $c \phi$ if $\lambda$ is small - that is, if $G$ and $H$ are large. Thus (i), (iii), and (iv) are satisfied. Condition (ii) can be achieved by choosing $c$ small.) Hypothesis (0.5) is a simplified version of hypothesis (2.8).

If $(0.5)$ holds, we can restart the iteration by setting $w_{k}^{1}=b_{k}-V F_{k}$, and $w_{k}^{j+1}=V\left[g_{k}-J_{k}\left(w_{1}^{j}, \ldots, w_{n}^{j}\right)\right]$ to obtain $a_{k} \leq w_{k}^{2} \leq w_{k}^{4} \leq \cdots \leq w_{k}^{3} \leq w_{k}^{1} \leq b_{k}$. So $w_{k}^{2 j}$ increases to $a_{k}^{\prime} \geq a_{k}$ and $w_{k}^{2 j+1}$ decreases to $b_{k}^{\prime} \leq b_{k}$. Clearly it may happen again that we obtain $a_{k}^{\prime}<b_{k}^{\prime}$ for some $k$. At this point, the reader will realize that we need to apply a transfinite induction argument to show that we must restart the procedure at most countably many times. We show in the transfinite induction argument that each time we restart, we can use the functions $c F_{1}, c F_{2}, \ldots, c F_{n}$, where $c$ is a suitably small positive constant. To summarize, if we assume $(0.2)$, $(0.3),(0.4)$, and $(0.5)$, then there are nonnegative functions $u_{1}, u_{2}, \ldots, u_{n}$ solving $(0.1)$. 
EXAMPLE. Let us look at a concrete example in $\mathbf{R}^{1}$ to show that the hypotheses can be easily verified in some situations. Let $E=(0, S) \subset \mathbf{R}^{1}$. Fix positive numbers $p, q, r$, and $s$ so that $p+q \geq 1$ and $r+s \geq 1$, and consider the system (with Dirichlet boundary conditions):

$$
-u^{\prime \prime}+c\left((u+v) / 2+u^{p} v^{q}\right)=1, \quad-v^{\prime \prime}+c\left((u+v) / 2+u^{r} v^{s}\right)=1 .
$$

As discussed above, we set $u_{1}=v_{1}=V 1$, and

$$
u_{2}=V\left[1-c\left(V 1+(V 1)^{p+q}\right)\right], \quad v_{2}=V\left[1-c\left(V 1+(V 1)^{r+s}\right)\right] .
$$

What very simple conditions on $S$ and $c$ can we impose to guarantee that the iterative method outlined above yields a solution? First, it would be ideal if $u_{2} \geq 0$ and $v_{2} \geq 0$ everywhere on $E$. One way to guarantee this is to require $V 1 \leq 1,1 \geq$ $2 c V 1 \geq c\left(V 1+(V 1)^{p+q}\right)$, and $1 \geq 2 c V 1 \geq c\left(V 1+(V 1)^{r+s}\right)$. Since $V 1(x)=$ $x(S-x) / 2, V 1 \leq 1$ provided $S \leq 2^{3 / 2}$. We also have $1 \geq 2 c V 1$ if $c \leq 4 / S^{2}$. These conditions suffice to guarantee that $a_{1}>0$ and $a_{2}>0$. To restart the iteration (if necessary), we will take $F_{k}$ to be a multiple of the first eigenfunction of $-\Delta$ (with Dirichlet boundary conditions) on $(e, S-e)$ for some small $e>0$. Note that we may take $\rho_{k k}=c / 2$. In order to satisfy (0.5iii), we need $c / 2$ to be larger then the first eigenvalue of $-\Delta$ on $(0, S)$ (and this is $\pi / S$ ). The two conditions needed for $c$ are: $4 / S^{2} \geq c>2 \pi / S$. Thus we require $S<4 / 2 \pi$. If these conditions are satisfied, the iterative procedure leads to a solution of the given system. Of course, these conditions can be relaxed considerably (by doing a considerably more detailed analysis).

In order to treat $(0.1)$ with general elliptic operators and measures, we depend heavily on using potential theory as it is formulated in the probabilistic potential theory literature. We recall most of what the reader needs to know in the text; Chapter VI of $[2]$ is a good reference. In $\S 1$, we introduce the potential theory framework. In particular, this is necessary to drop hypothesis $(0.3)$. In $\S 2$, we discuss analogues in the general situation of the hypotheses we introduced in this section, and we show how to solve (0.1). The main result is (2.9). The hypotheses look a bit forbidding when first encountered, but they are actually quite natural, as we have tried to indicate, and we discuss them further in $\S 3$. We also give another version of (2.9) in (3.1), where we drop a hypothesis (by replacing it with several more!). In $\S 4$, we briefly indicate some extensions. The appendix is devoted to a technical result necessary to show $b_{k}>0$. All Markov processes are confined to the appendix.

ACKNOWledgment. I would like to thank P. J. McKenna and M. Rao for several stimulating conversations on the subjects contained in this article.

NOTATION. In general, notation is standard and can be found, for example, in Chapter VI of [2]. We introduce most of it as needed. If $\mathcal{E}$ is any $\sigma$-algebra, then we use the same letter $\mathcal{E}$ to denote the collection of all $\mathcal{E}$-measurable functions which are real-valued. If $D$ is any collection of functions, then $\mathrm{b} D$ (resp. $\mathrm{p} D$ ) is the collection of bounded (resp. positive) functions in $D$. Thus $\mathrm{pb} \varepsilon$ is the collection of positive and bounded $\mathcal{E}$-measurable real-valued functions. Let $(A, \mathcal{A}, \mu)$ be a measure space, let $(B, B)$ be a measurable space, and let $\Phi: A \rightarrow B$ be measurable. We denote the image measure of $\mu$ under $\Phi$ by $\Phi(\mu)$; that is, $\Phi(\mu)(G)=\mu\left(\Phi^{-1}(G)\right)$ for every $G \in B$. A measure putting all of its mass at the point $x$ will be denoted by $\varepsilon_{x}$. 
1. The potential theory framework. Let $E$ be an open domain in $\mathbf{R}^{d}$, and let $\mathcal{E}$ be its Borel field. For each $k$ with $1 \leq k \leq n$, let $P_{t}^{k}$ be a sub-Markov semigroup on $(E, \mathcal{E})$, and let $U_{k}^{q}$ be its resolvent. (That is, for each $x \in E, P_{t}^{k}(x, \cdot)$ is a sub-probability measure on $(E, \mathcal{E})$. If we define $P_{t}^{k} f(x)=\int_{E} f(y) P_{t}^{k}(x, d y)$, then $P_{t}^{k} P_{s}^{k} f(x)=P_{s+t}^{k} f(x)$. The resolvent is $U_{k}^{q} f(x)=\int_{0}^{\infty} e^{-q t} P_{t}^{k} f(x) d t$.)

Notation. $C(E)$ is the collection of all continuous functions on $E . C_{c}(E)$ (resp. $C_{c}^{2}(E)$ ) is the collection of all continuous functions on $E$ with compact support in $E$ (resp. and having two continuous derivatives). $C_{0}(E)$ is the collection of functions in $C(E)$ vanishing at the boundary of $E$. We let $\mathrm{b} \mathcal{E}_{c}$ be the collection of bounded $\mathcal{E}$-measurable functions vanishing off a compact set contained in $E$.

We assume:

For each $q>0, U_{k}^{q}: \mathrm{b} \mathcal{E}_{c} \rightarrow C_{0}(E)$ and $\lim _{q \rightarrow \infty} q U_{k}^{q} f=f$ whenever $f \in C_{c}(E)$.

(reference measure) There is a Radon measure $m_{k}$ on $(E, \mathcal{E})$ so that $U_{k}^{q}(x, \cdot)<<m_{k}$ for each $x$ in $E$.

(duality) There is another sub-Markov semigroup $R_{t}^{k}$ on $(E, \mathcal{E})$ with resolvent $W_{k}^{q}$ so that

$$
\int_{E}\left(R_{t}^{k} f\right) g d m_{k}=\int_{E} f\left(P_{t}^{k} g\right) d m_{k}
$$

for every $f$ and $g$ in $\mathrm{p} \mathcal{E}$. For each $q>0, W_{k}^{q}: \mathrm{b} \mathcal{E}_{c} \rightarrow C_{0}(E)$ and $\lim _{q \rightarrow \infty} q W_{k}^{q} f=f$ whenever $f \in C_{c}(E)$.

(infinitesimal generators) There are linear operators $L_{k}$ and $L_{k}^{*}$ with domains $D_{k} \supset C_{c}^{2}(E)$ and $D_{k}^{*} \supset C_{c}^{2}(E)$ so that for every $f$ in $C_{c}^{2}(E),\left(f-P_{t}^{k} f\right) / t$ converges boundedly to $-L_{k} f$ as $t$ tends to zero, and $\left(f-R_{t}^{k} f\right) / t$ converges boundedly to $-L_{k}^{*} f$ as $t$ tends to zero. In addition, $L_{k}: C_{c}^{2}(E) \rightarrow \mathrm{b} \mathcal{E}_{c}$ and $L_{k}^{*}: C_{c}^{2}(E) \rightarrow \mathrm{b} \mathcal{E}_{c}$.

These conditions are satisfied by a huge class of strong Feller semigroups, the best known of which has $L_{k}=\Delta / 2$ with Dirichlet boundary conditions. In this case, $P_{t}^{k}$ is the semigroup of a Brownian motion "killed" when it reaches the boundary of $E$. This example also satisfies the additional hypothesis (1.5) below if $E$ is connected. There is also a large class of semigroups satisfying these conditions for which $L_{k}$ and $L_{k}^{*}$ are nonlocal integrodifferential operators.

DEFINITION. A function $f \in \mathrm{p} \mathcal{E}$ is said to be excessive for a resolvent $\left(V^{\alpha}\right)$ on $(E, \mathcal{E})$ if $\alpha V^{\alpha} f \leq f$ for every $\alpha>0$ and if $\lim _{\alpha \rightarrow \infty} \alpha V^{\alpha} f=f$.

As a consequence of the numbered assumptions above, there is a function $u_{k}(x, y)$ $\in \mathrm{p} \mathcal{E} \times \mathcal{E}$ so that:

(i) $x \rightarrow u_{k}(x, y)$ is excessive for $U_{k}^{q}$;

(ii) $y \rightarrow u_{k}(x, y)$ is excessive for $W_{k}^{q}$;

(iii) $U_{k}^{0} f(x)=\int_{E} u_{k}(x, y) f(y) m_{k}(d y)$ for every $f \in \mathrm{p} \mathcal{E}$;

(iv) $W_{k}^{0} f(y)=\int_{E} u_{k}(x, y) f(x) m_{k}(d x)$ for every $f \in \mathrm{p} \mathcal{E}$;

(v) for each $y \in E, x \rightarrow u_{k}(x, y)$ is lower semicontinuous;

(vi) for each $x \in E, y \rightarrow u_{k}(x, y)$ is lower semicontinuous. 
For the construction of this function, see Chapter VI of [2]. This is the precise version of the "Greeen function" we need to define potentials of measures. We assume

$$
u_{k}(x, y)>0 \quad \text { for every } x, y \in E .
$$

DEFINITION. If $\mu$ is a positive measure on $(E, \mathcal{E})$ then the potential of $\mu$ is defined to be $U_{k} \mu(x)=\int_{E} u_{k}(x, y) \mu(d y)$. If $\rho=\mu-\nu$ is the difference of the two positive measures $\mu$ and $\nu$, and if $U_{k}(\mu+\nu)<\infty$ a.e. $\left(m_{k}\right)$, then we set

$$
\begin{aligned}
U_{k} \rho(x) & =U_{k} \mu(x)-U_{k} \nu(x) \quad \text { on }\left\{U_{k}(\mu+\nu)<\infty\right\} \\
& =\infty \text { on }\left\{U_{k}(\mu+\nu)=\infty\right\} .
\end{aligned}
$$

The following properties are implied by $(1.4 \mathrm{v}, \mathrm{vi})$ and $(1.5)$ :

(i) If $K$ is compact in $E$, then $\inf \left\{u_{k}(x, y): x \in K\right\}>0$, and $\inf \left\{u_{k}(x, y): y \in K\right\}>0$.

(ii) If $\rho$ is a signed measure on $(E, \mathcal{E})$ with $U_{k}|\rho|<\infty$ a.e. $\left(m_{k}\right)$, then $U_{k} \rho$ is locally integrable. That is, $\int_{K} U_{k} \rho(x) m_{k}(d x)<\infty$ whenever $K$ is compact in $E$.

See [2].

Our purpose in this section is to develop a bit of potential theory necessary to formulate conditions sufficient to insure the existence of solutions for the following system of semilinear equations:

$$
\begin{aligned}
& -L_{1} u_{1}+f_{1}\left(u_{1}, \ldots, u_{n}\right)=\mu_{1}, \\
& -L_{n} u_{n}+f_{n}\left(u_{1}, \ldots, u_{n}\right)=\mu_{n} .
\end{aligned}
$$

Here, each $\mu_{k}$ is a positive measure on $(E, \mathcal{E})$ and each $f_{k}: \mathbf{R}^{n} \rightarrow \mathbf{R}$. Our analysis of the problem depends on adopting the following perspective, which is useful in potential theory.

Let $E_{1}, E_{2}, \ldots, E_{n}$ be $n$ distinct copies of $E$, and let $F=\bigcup_{k=1}^{n} E_{k}$. In general, we will denote a point of $E$ by $x$ (without a subscript) and a point in $E_{k}$ by $x_{k}$ (the subscript indicating in which copy of $E$ the point lies). Let $e_{k}: E \rightarrow E_{k}$ be the injection $e_{k} x=x_{k}$. Let $d_{E}$ be a metric on $E$ compatible with the topology of $E$. Define a metric $d_{F}$ on $F$ by setting

$$
\begin{aligned}
d_{F}\left(x_{k}, y_{j}\right) & =d_{E}(x, y) \quad \text { if } k=j \\
& =1 \quad \text { otherwise, }
\end{aligned}
$$

whenever $x_{k}=e_{k} x$ and $y_{j}=e_{j} y$. This metric induces a topology on $F$ which obviously extends the topology on $E$. Its Borel field $\mathcal{F}$ is characterized by the fact that its trace on $E_{k}$ is $\mathcal{E}_{k}$, a copy of $\mathcal{E}$.

Define

$$
\mu=\sum_{k=1}^{n} e_{k}\left(\mu_{k}\right)
$$

and define a new sub-Markov semigroup $P_{t}$ on $F$ by setting

$$
P_{t} f\left(x_{k}\right)=\int_{E_{k}} f(y) e_{k}\left(P_{t}^{k}(x, d y)\right)
$$


whenever $x_{k}=e_{k} x$ and $f \in \mathrm{b} \mathcal{F}$. It is easy to check that $P_{t}$ is a semigroup on $F$, and it has infinitesimal generator

$$
L=\sum_{k=1}^{n} L_{k} 1_{E_{k}}
$$

and resolvent

$$
U^{q}=\int_{0}^{\infty} e^{-q t} P_{t} d t
$$

Note that $U^{q}\left(x_{k}, \cdot\right)=e_{k}\left(U_{k}^{q}(x, \cdot)\right)$ whenever $x_{k}=e_{k} x$. If we define the measure $M$ on $(F, \mathcal{F})$ by setting

$$
M=\sum_{k=1}^{n} e_{k}\left(m_{k}\right)
$$

then $U^{q}(z, \cdot) \ll M$ for every $z$ in $F$. The potential density $u(\cdot, \cdot)$ for $U^{0}$ is given by

$$
\begin{aligned}
u\left(x_{k}, y_{j}\right) & =\mu_{k}(x, y) & & \text { if } k=j, \\
& =0 & & \text { otherwise }
\end{aligned}
$$

for $x_{k}=e_{k} x$ and $y_{j}=e_{j} y$.

We find this perspective handy because, in solving (1.7), we need to analyze not the inverse of the linear system $\left(-L_{1},-L_{2}, \ldots,-L_{n}\right)$, but instead the inverse of the perturbed linear system

$$
\begin{aligned}
& -L_{1} u_{1}-\alpha_{11} u_{1}-\alpha_{12} u_{2}-\cdots-\alpha_{1 n} u_{n}, \\
& -L_{n} u_{n}-\alpha_{n 1} u_{1}-\alpha_{n 2} u_{2}-\ldots-\alpha_{n n} u_{n} .
\end{aligned}
$$

(The $\alpha_{k j}$ are positive functions on $E$ to be chosen in $\S \S 2$ and 3 . In general, they may be unbounded and have infinities.) We may obtain the inverse of this system by perturbing the resolvent $U^{q}$ as follows. Define a kernel $B(z, d y)$ from $(F, \mathcal{F})$ to $(F, \mathcal{\exists})$ : for $1 \leq k \leq n$, set

$$
B\left(x_{k}, \cdot\right)=\sum_{j=1}^{n} \alpha_{k j}(x) \varepsilon_{x_{j}}(\cdot),
$$

where $x_{j}=e_{j} x$. For each $q \geq 0$, define a new kernel on $(F, \mathcal{F})$ by setting

$$
V^{q}=\sum_{k=0}^{\infty}\left(U^{q} B\right)^{k} U^{q} .
$$

The infinite sum makes sense since each term is positive. It is easy to check that $V^{q}$ satisfies the resolvent equation: if $p \geq 0$ and $q \geq p$, then $V^{p}=V^{q}+(q-p) V^{p} V^{q}$. If the $\alpha_{k j}$ are too large, then $V^{0}(z, \cdot)$ may not be $\sigma$-finite for some $z$ in $F$. We always assume:

(1.13) There is a strictly positive function $\Psi \in \mathcal{F}$ so that $V^{0} \Psi(z)<\infty$ for every $z$ in $F$.

If $f \in \mathrm{p} \mathcal{F}$, an application of the resolvent equation shows that $V f$ is excessive. Since $V^{q}$ is a resolvent and we are assuming (1.13), we have the following maximum principle. 
(1.14) Proposition [9,IX,T68]. Let $f \in \mathrm{p} \mathcal{F}$. If $h$ is an excessive function for $\left(V^{q}\right)$ and if $V f \leq h$ on $\{f>0\}$, then $V f \leq h$ on $F$.

(1.15) Notation. $V(x, \cdot) \equiv V^{0}(x, \cdot)$.

This perturbation $V^{q}$ of $U^{q}$ is well understood in the classical case when the $a_{k j}$ and $U^{q}$ are well behaved.

(1.16) Proposition [3]. Assume $F$ is locally compact with a countable base and that $P_{t}$ is a Feller semigroup with strong infinitesimal generator $(L, D(L))$ on $C_{0}(F)$. If $B$ is a continuous operator on $C_{0}(F)$, then $V^{q}$ is the resolvent of a strong Feller semigroup on $C_{0}(F)$ with infinitesimal generator $(L+B, D(L))$.

We will work without such strong hypotheses. In particular, $P_{t}$ may not be Feller and $B$ is usually not continuous.

(1.17) Definition. Set

$$
v(z, y)=\sum_{k=0}^{\infty}\left[(U B)^{k} u(\cdot, y)\right](z)=\sum_{k=0}^{\infty} \int_{F} u(w, y)(U B)^{k}(z, d w) .
$$

If $\rho$ is a positive or signed measure on $F$, then the potential of $\rho, V \rho$, is defined with the density $v$ just as $U \mu$ was defined with the density $u$. In $\S 2$, hypothesis (2.1c) will imply $\alpha_{k j}>0$, and this in turn implies $v(z, y)>0$.

(1.19) LEMMA. (i) If $\rho$ is a signed measure on $(F, \mathcal{F})$ with $V|\rho|<\infty$ a.e. $(M)$, then $\int_{K} V \rho d M<\infty$ whenever $K \subset F$ is compact. That is, $V \rho$ is locally integrable.

(ii) Assume (2.1c). If $K \subset F$ is compact, then $\inf \{v(z, y): y \in K\}>0$ for every $z$ in $F$ and $\inf \{v(z, y): z \in K\}>0$ for every $y$ in $F$.

Proof . (i) $V|\rho|=U\left(|\rho|+\sum_{k=0}^{\infty} B(U B)^{k} U|\rho|\right)$. Since the right-hand side is the $U$-potential of a positive measure, it must be locally integrable by (1.6ii) and the definition of $U$. Since $V|\rho|$ is locally integrable, $V \rho$ is locally integrable.

(ii) This follows from (1.6i) and (2.1c) since $v(x, y) \geq u(x, y)$. Q.E.D.

(1.20) Proposition. Let $\rho$ be a signed measure on $(F, \mathcal{F})$ so that $V|\rho|<\infty$ a.e. $(M)$. Then $(L+B) V \rho=-\rho$ in the sense that

$$
\int_{F}\left(L^{*} f\right) V \rho d M+\int_{F} f(B V \rho) d M=-\rho(f)
$$

whenever $f \in C_{c}^{2}(F)$, and where

$$
L^{*}=\sum_{k=1}^{n} L_{k}^{*} 1_{E_{k}}
$$

Proof. Assume first that $\rho$ is a positive measure on $(F, \mathcal{F})$ and let $f \in \mathrm{p} C_{c}^{2}\left(E_{k}\right)$. By (1.19), $V \rho$ is locally integrable. Since the support of $L_{k}^{*}$ is compact in $E_{k}$ by hypothesis (1.3), the following integrals are well defined and we have

$$
\int_{E_{k}}\left(L_{k}^{*} f\right) V \rho d M=\int_{E_{k}}\left(L_{k}^{*} f\right) \sum_{j=0}^{\infty}(U B)^{j} U \rho d M .
$$


Since the integrands are absolutely integrable, Fubini's theorem applies, and we may rewrite $(1.22)$ as

$$
\int_{E_{k}}\left(L_{k}^{*} f\right) U \rho d M+\sum_{j=1}^{\infty} \int_{E_{k}}\left(L_{k}^{*} f\right)(U B)^{j} U \rho d M .
$$

To analyze (1.23), we need the following result.

(1.24) LEMMA. If $\gamma$ is a positive measure on $(E, \mathcal{E})$ with $U_{k} \gamma<\infty$ a.e. $\left(m_{k}\right)$, then

$$
\int_{E}\left(L_{k}^{*} f\right) U_{k} \gamma d m_{k}=-\gamma(f)
$$

whenever $f \in C_{c}^{2}(E)$.

ProOF. Recall that $W_{k} L_{k}^{*} f=W_{k}\left[\lim _{t \rightarrow 0}\left(R_{t}^{k} f-f\right) / t\right]=\lim _{t \rightarrow 0} W_{k}\left(R_{t}^{k} f-f\right) / t$ since the convergence is bounded by hypothesis (1.3). But this last term may be rewritten as

$$
-\lim _{t \rightarrow 0} t^{-1} \int_{0}^{t} R_{s}^{k} f d s=-f .
$$

Since

$$
\int_{E}\left|L_{k}^{*} f\right| U_{k} \gamma d m_{K}<\infty
$$

Fubini's theorem applies to the first term in (1.25) and yields

$$
\int_{E} W_{k} L_{k}^{*} f d \gamma=-\gamma(f) \text { Q.E.D. }
$$

Thus the first term in (1.23) may be rewritten as $-\rho(f)$, while the infinite sum in (1.23) may be rewritten as

$$
\sum_{j=1}^{\infty} \int_{E_{k}}\left(L_{k}^{*} f\right) U(B U)^{j} \rho d M=-\sum_{j=1}^{\infty} \int_{E_{k}} f(B U)^{j} \rho d M .
$$

Since $f \geq 0$ and $(B U)^{j} \rho \geq 0$, we may rewrite this as

$$
-\int_{E_{k}} f \sum_{j=1}^{\infty}(B U)^{j} \rho d M=-\int_{E_{k}} f(B V \rho) d M
$$

This proves (1.21) for $\rho \geq 0$ and $f \in \mathrm{p} C_{c}^{2}\left(E_{k}\right)$. The general case follows by linearity. Q.E.D.

Now how can this potential theory aid us in solving (1.7)? Formally, we may rewrite (1.7) as

$$
\begin{aligned}
& -L_{1} u_{1}-\alpha_{11} u_{1}-\cdots-\alpha_{1 n} u_{n}=\mu_{1}-G_{1}\left(x, u_{1}, \ldots, u_{n}\right), \\
& -L_{n} u_{n}-\alpha_{n 1} u_{1}-\cdots-\alpha_{n n} u_{n}=\mu_{n}-G_{n}\left(x, u_{1}, \ldots, u_{n}\right),
\end{aligned}
$$

where $G_{k}\left(x, u_{1}, \ldots, u_{n}\right)=\alpha_{k 1}(x) u_{1}+\cdots+\alpha_{k n}(x) u_{n}+f_{k}\left(u_{1}, \ldots, u_{n}\right)$. Define a function $H: F \times \mathbf{R}^{n} \rightarrow \mathbf{R}$ by setting $H\left(x_{k}, \mathbf{u}\right)=G_{k}\left(x, u_{1}, \ldots, u_{n}\right)$ for $x_{k}=e_{k} x$. If 
$u: F \rightarrow \mathbf{R}$, let $G\left(x_{k}, u\right)=H\left(x_{k}, u\left(x_{1}\right), u\left(x_{2}\right), \ldots, u\left(x_{n}\right)\right)$ where $x_{j}=e_{j} x$. If we try to solve (1.26) by inverting, we find we need to solve the following equation for $u$ :

$$
u=V[\mu-G(\cdot, u(\cdot))],
$$

where $\mu$ and $V$ are defined above. Once we find such a function $u$, Proposition (1.20) shows that $-(L+B) u=\mu-G(\cdot, u(\cdot))$. Thus, if we set $u_{1}(x)=u\left(e_{1} x\right), u_{2}(x)=$ $u\left(e_{2} x\right), \ldots, u_{n}(x)=u\left(e_{n} x\right)$, we have that the functions $u_{1}, u_{2}, \ldots, u_{n}$ solve (1.26), and so they solve (1.7).

2. The main argument. In this section, we develop hypotheses which allow us to solve the equation $u=V[\mu-G(\cdot, u(\cdot))]$. We assume the notation and hypotheses discussed in $\S 1$. (In particular, we assume (1.0) through (1.3), (1.5), and (1.13).) In addition, we assume the following conditions throughout this section.

(a) $\mu$ is a positive measure, $\mu(F)>0$ and $V \mu<\infty$ a.e. $(M)$.

(b) $G(x, u)$ is continuous in $u$, and $G(x, 0)=0$.

(c) The functions $\alpha_{k j}$ have been chosen strictly positive so that $G(x, u)<G(x, v)$ whenever $0 \leq u(\cdot)<v(\cdot) \leq V \mu(\cdot)$.

Recall (2.1c) implies $v(z, y)>0$ for every $z$ and $y$ in $F$.

For most of this section, we work with a "cutoff" of $G, J(u)(z)$, defined as follows: for $x$ in $E$ and $x_{k}=e_{k} x$,

$$
J(u)\left(x_{k}\right)=G\left(x_{k}, u\right) 1_{\left\{u\left(e_{1} x\right) \geq 0, \ldots, u\left(e_{n} x\right) \geq 0\right\}} .
$$

(2.3) Proposition. Assume (2.1) and the following condition:

$$
M(V \mu>V G(V \mu))>0 .
$$

Then there are two functions $u_{1}$ and $v_{1}$ on $F$ so that $u_{1} \leq v_{1} \leq V \mu, u_{1}=$ $V\left[\mu-J\left(v_{1}\right)\right]$, and $v_{1}=V\left[\mu-J\left(u_{1}\right)\right]$.

Notation. If $w \in \mathcal{F}$, let $H(w)=\mu-J(w)$. We also adopt the convention $\infty-\infty=\infty$.

REMARK. By (2.4), $M(V G(V \mu)<\infty)>0$. This implies $V G(V \mu)<\infty$ a.e. $(M)$. For if $M(V G(V \mu)=\infty)>0$, then we would have $\alpha V^{\alpha}(V G(V \mu))=\infty$ since $v^{\alpha}(z, \cdot)$ is bounded away from zero on compacts.

PROOF. Set $w_{1}=V \mu$, and define $w_{2}=V H\left(w_{1}\right)$. Note that $w_{2}$ is well defined a.e. $(M)$ since $V \mu<\infty$ a.e. $(M)$ and $J \geq 0$. By $(2.4), M\left(w_{2}>0\right)>0$. Since $J \geq 0$, $w_{2} \leq w_{1}$. Now set $w_{3}=V H\left(w_{2}\right)$ : once again, this is well defined a.e. $(M)$. Since $w_{2} \leq w_{1}, J\left(w_{2}\right) \leq J\left(w_{1}\right)$ by $(2.1 \mathrm{c})$ and (2.2). Thus $w_{3} \geq w_{2}$. Since $J\left(w_{2}\right) \geq 0$, $w_{3} \leq V \mu=w_{1}$. We have proved that $w_{2} \leq w_{3} \leq w_{1}$. This alternating or oscillating behavior continues. By induction, define $w_{k+1}=V H\left(w_{k}\right)$. Note that $w_{k+1}$ is well defined a.e. $(M)$.

(2.5) Lemma. Assume $w_{2} \leq \cdots \leq w_{2 k} \leq w_{2 k+1} \leq \cdots \leq w_{1}$. Then $w_{2 k} \leq$ $w_{2 k+2} \leq w_{2 k+3} \leq w_{2 k+1}$.

PROOF. The lemma follows immediately from these observations:

(i) $J\left(w_{2 k+1}\right) \leq J\left(w_{2 k-1}\right)$ implies $w_{2 k+2} \geq w_{2 k}$.

(ii) $J\left(w_{2 k+1}\right) \geq J\left(w_{2 k}\right)$ implies $w_{2 k+2} \leq w_{2 k+1}$.

(iii) $J\left(w_{2 k+2}\right) \leq J\left(w_{2 k+1}\right)$ implies $w_{2 k+3} \geq w_{2 k+2}$.

(iv) $J\left(w_{2 k+2}\right) \geq J\left(w_{2 k}\right)$ implies $w_{2 k+3} \leq w_{2 k+1}$. Q.E.D. 
Therefore, the sequence $\left(w_{2 k}\right)$ increases to a function $u_{1}$, while the sequence $\left(w_{2 k+1}\right)$ decreases to a function $v_{1}$ with $-\infty<u_{1} \leq v_{1}<V \mu$ a.e. $(M)$. (Note that (2.4) and (1.5) imply strict inequality in $v_{1}<V \mu$.) Since $w_{2 k+1}=V \mu$ $V J\left(w_{2 k}\right)$, the monotone convergence theorem yields $v_{1}=V \mu-V J\left(u_{1}\right)$. Since $J\left(w_{1}\right) \geq J\left(w_{2 k+1}\right)$ and $V J\left(w_{1}\right)<\infty$ a.e. $(M)$ by (2.4), we may apply the dominated convergence theorem to the equality $w_{2 k+2}=V \mu-V J\left(w_{2 k+1}\right)$ to obtain $u_{1}=V \mu-V J\left(v_{1}\right)$. Q.E.D.

The measure $\mu$ can always be decomposed as $r \cdot d M+\mu^{s}$, where $\mu^{s}$ is singular with respect to $M$ and $r \geq 0$. Assume

$$
r>0 \text { on } F \text {. }
$$

(2.7) LEMMA. Let $a$ and $b$ be two functions on $F$ so that $u_{1} \leq a \leq b \leq V \mu$, $M(b>a)>0$, and suppose that $a=V \mu-V J(b)$ and $b=V \mu-V J(a)$. Then $b-a>0$ (remember $\infty-\infty=\infty)$ and $b>0$ on $F$.

Proof. By hypothesis, $M(b-a=V[J(b)-J(a)]>0)>0$, and $b \geq a$, so $M(J(b)>J(a))>0$ and $J(b) \geq J(a)$. Therefore, $b-a=V[J(b)-J(a)]>0$ since $v(\cdot, \cdot)>0$. Since $b+V J(a)=V \mu$, we have that $V J(a) \leq V \mu$ on $\{b>0\}$. Thus we have $V J(a) \leq V \mu$ on $\{a>0\} \supset\{J(a)>0\}$ since $a \leq b$. By (1.14), $V J(a) \leq V \mu$ on $F$. Thus $b$ must be nonnegative. Now assume $\{b=0\}$ is nonempty, and let $z \in\{b=0\}$. Then we have $V \mu(z)=V J(a)(z)<\infty$. Since $b-a>0, a(z)<0$ and $V J(b)(z)>V \mu(z)$. Note that $\{V J(b)>V \mu\}$ is a finely open set (for the fine topology generated by the resolvent $\left.\left(V^{\alpha}\right)\right)$. By (A.1) and (2.6), $V \mu(w)<V J(a)(w)$ for some $w$ in $F$. But this contradicts $b \geq 0$. Therefore, $\{b=0\}=\varnothing$. Q.E.D.

The next hypothesis will be used in a crucial way in the main theorem in this section. It will be discussed further in the remark following it and also in $\S 3$. See also the discussion in the introduction. Assume:

(a) There are positive functions $\rho_{k j}: F \rightarrow \mathbf{R}$ so that

(i) $J(c+\gamma)\left(x_{k}\right)-J(c)\left(x_{k}\right) \geq \sum_{j=1}^{n} \rho_{k j}\left(x_{j}\right) \gamma\left(x_{j}\right)$ a.e. $(M)$ when-

ever $x_{j}=e_{j} x$ and $c$ and $\gamma$ are functions with $u_{1}^{+} \leq c \leq c+\gamma \leq v_{1}$;

(ii) Whenever $K \subset E_{k}$ is compact, $\inf \left\{\rho_{k k}(z): z \in K\right\}>0$.

(NotATION: Let $\Phi(\gamma)\left(x_{k}\right)=\sum_{j=1}^{n} \rho_{k j}\left(x_{j}\right) \gamma\left(x_{j}\right)$.)

(b) There is a nonnegative bounded function $f$ on $F$ and numbers $\delta>0$ and $\pi>0$ so that

(i) the set $\{f>0\}$ is contained in a compact set $K_{f}$ and $K_{f} \subset$ $\left\{u_{1}>\delta\right\} \cap\left\{v_{1}<\pi\right\} ; M(f>0)>0$.

(ii) $f \leq J\left(v_{1}-V f\right)-J\left(u_{1}\right)$.

(iii) $f \leq \Phi\left(V\left[\Phi(V f) 1_{\left\{u_{1}>V f\right\}}\right]\right)$.

REMARK. Condition $(2.8 \mathrm{~b}$ (iii)) is the difficult one to fulfill, consistent with achieving (1.13). Let us show that $(2.8 \mathrm{~b}(\mathrm{i}))$ and $(2.8 \mathrm{~b}(\mathrm{ii}))$ can always be achieved. If $u_{1}<v_{1}$, we may choose $\delta>0$ and $\pi>0$ so that $M\left(u_{1}>\delta, v_{1}<\pi\right)>0$. Let $g$ be any bounded nonnegative function so that $\{g>0\}$ has compact closure in $F$ contained in $\left\{u_{1}>\delta\right\} \cap\left\{v_{1}<\pi\right\}, V g$ is everywhere finite on $F, M(g>0)>0$ and $J\left(v_{1}\right)-J\left(u_{1}\right) \geq g$. Let $0<p<1$. Then $\left|J\left(v_{1}-V p g\right)-J\left(u_{1}\right)\right| \geq \Phi\left(v_{1}-u_{1}-V p g\right)$ on $\left\{v_{1}-u_{1} \geq V p g\right\}$. Recall that $v_{1}-u_{1}=V\left[J\left(v_{1}\right)-J\left(u_{1}\right)\right]$. Since $(1.6,2.1 \mathrm{c})$ imply $\inf \left\{\left(v_{1}-u_{1}\right)(z): z \in\{g>0\}\right\}>0$, we also have that $\inf \left\{\left(J\left(v_{1}\right)-J\left(u_{1}\right)\right)(z): z \in\right.$ $\{g>0\}\}=c>0$. If we choose $p$ so small that $p g<J\left(v_{1}\right)-J\left(u_{1}\right)$ on $\{g>0\}$, 
we have that $\Phi\left(v_{1}-u_{1}-V p g\right)>d>0$ on $\{g>0\}$ since $\inf \left\{\rho_{k k}(z): z \in\{g>\right.$ $\left.0\} \cap E_{k}\right\}>0,1 \leq k \leq n$. Thus, by taking an even smaller $p$ so that $p g<d$, we obtain $p g \leq J\left(v_{1}-V p g\right)-J\left(u_{1}\right)$, and we set $f=p g$.

(2.9) Theorem. Assume (2.1), (2.4), (2.6), and (2.8). There is a function $u$ so that $0 \leq u \leq V \mu$ and $u=V[\mu-G(\cdot, u(\cdot))]$.

PROOF. The heart of this proof is a transfinite induction argument. We construct two collections of functions $\left(u_{\gamma}\right) \subset \mathcal{F}$ and $\left(v_{\gamma}\right) \subset \mathcal{F}$ : they are indexed by the ordinals $\gamma \geq 1$.

Notation. Set $a_{1}=-\infty$ and $b_{1}=V \mu$. For every ordinal $\beta \geq 2$, set $a_{\beta}=$ $\sup \left\{u_{\gamma}: 1 \leq \gamma<\beta\right\}$, and set $b_{\beta}=\inf \left\{v_{\gamma}: 1 \leq \gamma<\beta\right\}$.

For each ordinal $\beta \geq 1$, define the proposition $\phi(\beta)$ to be the statement:

$$
\begin{aligned}
& \text { If } M\left(a_{\beta}<b_{\beta}\right)>0, \text { then there are functions } u_{\beta} \text { and } v_{\beta} \text { so that } \\
& a_{\beta} \leq u_{\beta} \leq v_{\beta} \leq b_{\beta} \leq V \mu, M\left(v_{\beta}<b_{\beta}\right)>0, u_{\beta}=V\left[\mu-J\left(v_{\beta}\right)\right] \\
& \text { and } v_{\beta}=V\left[\mu-J\left(u_{\beta}\right)\right] \text {. }
\end{aligned}
$$

We have already verified the truth of $\phi(1)$ in Proposition (2.3). Let us assume $\phi(\gamma)$ is true for every $\gamma<\beta$, and prove $\phi(\beta)$ is true. Since $b_{\gamma}=V \mu-V J\left(a_{\gamma}\right)$ for every $\gamma<\beta$, the monotone convergence theorem lets us conclude that $b_{\beta}=$ $V \mu-V J\left(a_{\beta}\right)$. Similarly, the dominated convergence theorem lets us conclude that $a_{\beta}=V \mu-V J\left(b_{\beta}\right)$. Set $a=a_{\beta}$ and $b=b_{\beta}$, and assume $M(a<b)>0$. By $(2.7)$, $b>a$ and $b>0$ on $F$.

Let $f$ be the function chosen in $(2.8 \mathrm{~b})$. We now show that for some $p$ with $0<p<1, p f$ satisfies the conditions in (2.8b) if we replace $u_{1}$ with $a$ and $v_{1}$ with $b$. (We essentially repeat the argument given in the remark following (2.8).)

First note that $\{p f>0\} \subset\left\{u_{1}>\delta, v_{1}<\pi\right\} \subset\{a>\delta, b<\pi\}$, and $M(p f>0)>0$. Second, since $\inf \{(b-a)(z)=V[J(b)-J(a)](z): z \in\{p f>$ $0\}\}>0, p$ can be chosen small so that $J(b)-J(a)>p f$ on $\{f>0\}$. Thus $b-a-V p f>c>0$ on $\{p f>0\}$. It follows that $\Phi(b-a-V p f)>d>0$ on $\{p f>0\}$ since $\inf \left\{\rho_{k k}(z): z \in\{p f>0\} \cap E_{k}\right\}>0,1 \leq k \leq n$. We may take an even smaller $p$ so that $\Phi(b-a-V p f)>p f$ and we obtain $p f \leq J(b-V p f)-J(a)$. Third, and finally, we show that $p f \leq \Phi\left(V\left[\Phi(V p f) 1_{\{a>V p f\}}\right]\right)$. Multiplying $(2.8 \mathrm{~b}(\mathrm{iii}))$ by $p$ yields $p f \leq \Phi\left(V\left[\Phi(V p f) 1_{\left\{u_{1}>V f\right\}}\right]\right)$. Since $\left\{u_{1}>V f\right\} \subset\{a>V p f\}$, we have the result.

Set $\varepsilon=V p f, w_{1}=b-\varepsilon, w_{2}=V H\left(w_{1}\right)$, and $w_{3}=V H\left(w_{2}\right)$.

(2.11) LEMMA. $a \leq w_{2} \leq w_{3} \leq w_{1}$.

PROOF. Since $p f \leq J(b-V p f)-J(a), V[J(b)-J(a)] \geq \varepsilon$, or $b-\varepsilon \geq a$. Thus $w_{1} \geq a$. To obtain $w_{2} \geq a$, we need $V H(b-\varepsilon) \geq V H(b)$, or $V[J(b)-J(b-\varepsilon)] \geq 0$. This holds since $J$ is increasing and $b \geq b-\varepsilon$. To obtain $w_{2} \leq w_{1}$, we need $V H(a)-V H(b-\varepsilon) \geq \varepsilon$, or $V[J(b-\varepsilon)-J(a)] \geq V p f$. This holds since $p f \leq$ $J(b-V p f)-J(a)$. Since $w_{2} \leq w_{1}$, we obtain $w_{3} \geq w_{2}$. Finally, to obtain $w_{3} \leq w_{1}$, we need $V H\left(w_{2}\right) \leq b-\varepsilon$, or $V H(V H(b-\varepsilon)) \leq b-\varepsilon$. That is, we need $V[\mu-J(V H(b-\varepsilon))] \leq V[\mu-J(a)]-\varepsilon$, or $V J(a)+V p f \leq V J(V H(b-\varepsilon))$. To obtain this, we need only have $J(a)+p f \leq J(V H(b-\varepsilon))$, or $p f \leq$ $J(V H(b-\varepsilon))-J(a)=J(a+V[J(b)-J(b-\varepsilon)])-J(a)$. By (2.8a), it suffices to have $p f \leq \Phi(V[J(b)-J(b-\varepsilon)])$ on $\left\{u_{1}>0\right\} \subset\{a>0\}$. Applying (2.8a) again, we see 
it suffices to have $p f \leq \Phi\left(V\left[\Phi(V p f) 1_{\{b>V p f\}}\right]\right)$. Since $\{V p f<a\} \subset\{V p f<b\}$, we see that it suffices to have

$$
p f \leq \Phi\left(V\left[\Phi(V p f) 1_{\{V p f<a\}}\right]\right) .
$$

We showed before (2.11) that $f$ has this property. Q.E.D.

Now we can set $w_{k+1}=V H\left(w_{k}\right)$ as we did earlier in this section. The proof of Proposition (2.3) shows that we obtain functions $u_{\beta} \leq v_{\beta}$ so that $v_{\beta}=V \mu-V J\left(u_{\beta}\right)$ and $u_{\beta}=V \mu-V J\left(v_{\beta}\right)$ : we have verified $\phi(\beta)$. Choose a finite measure $N$ on $(F, \mathcal{F})$ which is equivalent to $M$ so that $N\left(v_{1}-u_{1}\right)<\infty$. The sequence $\pi(\beta)=N\left(v_{\beta}-u_{\beta}\right)$ is a positive and strictly decreasing sequence. Thus $\pi(\beta)=0$ for some countable ordinal $\beta$. That is, there is a $\beta$ so that $u_{\beta}=b_{\beta}=v_{\beta}=u$. But then $u=V \mu-V J(u)$. By (2.7), $u \geq 0$, so we have $u=V \mu-V G(\cdot, u(\cdot))$. This concludes the proof of (2.9). Q.E.D.

3. The final result and discussion of hypotheses. In this section, we again assume the notations and hypotheses of $\S 1$. This next result drops the hypothesis (2.6) (but adds some others !).

(3.1) THEOREM. Assume (2.1) and suppose there is a decreasing sequence of functions $h_{k}$ on $F$ so that

(i) $h_{k}>0$ on $F$;

(ii) $\lim _{k \rightarrow \infty} V h_{k}=0$;

(iii) $M\left(V\left(\mu+h_{k}\right)>V G\left(V\left(\mu+h_{k}\right)\right)\right)>0$.

Assume (2.8). (Note: the function $f$ may vary with $k$.) Then there is a function $u \geq 0$ with $u=V[\mu-G(\cdot, u(\cdot))]$.

Proof. By (iii), $V J V\left(\mu+h_{k}\right)=V G V\left(\mu+h_{k}\right)<\infty$ on a set of positive measure, and hence a.e. $(M)$. Let $\Phi=J\left(V\left(\mu+h_{1}\right)\right)$, so $V \Phi<\infty$ a.e. $(M)$. For each $k$ the hypotheses of Theorem (2.9) are satisfied, so there is a function $u_{k}$ with $0 \leq u_{k} \leq V\left(\mu+h_{k}\right)$ and $u_{k}=V\left[\mu+h_{k}-J\left(u_{k}\right)\right]$. Since $u_{k} \leq V\left(\mu+h_{k}\right), J\left(u_{k}\right) \leq \Phi$, so $c_{k}=J\left(u_{k}\right) / \Phi$ is a bounded sequence in $L^{\infty}(M)$. By Alaoglu's theorem, there is a subsequence $c_{k(l)}$ of $c_{k}$ and a function $w \geq 0$ so that $\int c_{k(l)} g d M$ converges to $\int w g d M$ for every $g \in L^{1}(M)$. For almost every $z, v(z, \cdot) \Phi(\cdot) \in L^{1}(M)$, so $V \Phi c_{k(l)}=V J\left(u_{k(l)}\right)$ converges to $V w$. Since $u_{k(l)}=V\left[\mu+h_{k(l)}-J\left(u_{k(l)}\right)\right], u_{k(l)}$ must converge (a.e.) to a function $u \geq 0$. Since $J\left(u_{k}\right) \leq \Phi$ and $V \Phi<\infty$ a.e. $(M)$, we may apply the dominated convergence theorem to conclude $\lim V J\left(u_{k(l)}\right)=$ $V J(u)$, and we have proved that $u=V[\mu-J(u)]$. Q.E.D.

Theorem (3.1) is perhaps not quite as satisfying as the result in $\S 2$ since $u$ is obtained by a compactness method instead of monotone approximation. One might hope that since the $h_{k}$ decrease, the $u_{k}$ should also decrease. This does not seem to be the case, however, and one must be careful to recall that $J$ is not a local function of its argument. For example, suppose we try to prove the $u_{k}$ decrease. By subtracting, we obtain $u_{k}-u_{k+1}+V\left[J\left(u_{k}\right)-J\left(u_{k+1}\right)\right]=V\left[h_{k}-h_{k+1}\right]$, so $V\left[J\left(u_{k}\right)-J\left(u_{k+1}\right)\right] \leq V\left[h_{k}-h_{k+1}\right]$ on $\left\{u_{k}>u_{k+1}\right\}$. At this point it is tempting to say $\left\{J\left(u_{k}\right)>J\left(u_{k+1}\right)\right\} \subset\left\{u_{k}>u_{k+1}\right\}$ (which is incorrect), so the maximum principle yields $V\left[J\left(u_{k}\right)-J\left(u_{k+1}\right)\right] \leq V\left[h_{k}-h_{k+1}\right]$ everywhere. This would lead to the (incorrect) conclusion that $u_{k}>u_{k+1}$.

We now discuss the hypotheses in $\S \S 1$ and 2 . 
Hypotheses (1.0) through (1.3), (1.5). These hypotheses are quite reasonable and cover a large class of elliptic and integrodifferential operators.

Hypothesis (1.13). The condition $V \Psi<\infty$ is necessary to insure we have a nontrivial potential theory: whether or not it is satisfied depends on how large the $\alpha_{k j}$ are.

Hypothesis (2.1). Parts (a) and (b) are simple requirements. Part (c) is the tricky one. Note that $V \mu$ depends on the $\alpha_{k j}$ : as the $\alpha_{k j}$ grow, so does $V \mu$. There are two situations in which this hypothesis might be easily satisfied. First, if $\partial f_{k} / \partial x_{j}>0$ for all $k$ and $j$, then the $\alpha_{k j}$ can be chosen to be zero. In this case, (1.13) is instantly satisfied. Or it may be the case that $\partial f_{k} / \partial x_{j}$ is bounded below, say by $\xi_{k j}$. If $\xi_{k j}<0$, set $\alpha_{k j}=-\xi_{k j}$; otherwise, set $\alpha_{k j}=0$. In this case, (1.13) may or may not be satisfied. In the general case, it is best to adopt the following philosophy. Having chosen $\alpha_{k j} \geq 0$ so that (1.13) holds, hypotheses (2.1), (2.4), and (2.8) delineate a class of measures $\mu$ for which (1.7) can be solved. It would be nice to have a deeper understanding of the relationships between (2.1) and (1.13).

Hypothesis (2.4). This is an interesting and suggestive one. If $V \mu \leq V G(V \mu)$ everywhere, it is known in probability and potential theory that $\mu$ is a balayage of $G(V \mu)$. It is easy to cite at least one condition forbidding this and so guaranteeing that (2.4) holds: if $\mu$ charges a polar set (i.e., a set contained in the infinities of an excessive function), then it cannot be the balayage of a function (and note that $G(V \mu)$ is a function).

Hypothesis (2.8). Part (a) is a simple requirement which can always be achieved by increasing the $\alpha_{k k}$ slightly if necessary. Part (b) is a nontrivial assumption. Parts (i) and (ii) can always be achieved, as is shown in the Remark following the hypothesis. Part (iii) is the hard part. Since $u_{1} \geq a$, it may sometimes be easier to check (iii) with $a$ replacing $u_{1}$. Notice that while hypotheses (2.1) and (2.4) seem pretty close to optimal for our presentation, hypothesis $(2.8)$ is really more than is needed. We present it in this form as an attempt to render a complicated result a bit more palatable. By following the proof of (2.9), the reader will see exactly what is needed.

4. Extensions. The methods and theorems we have discussed can be extended to parabolic semigroups. For example, $L_{k}$ could be $\partial / \partial t-\Delta$. Hypotheses (1.5) and (1.6i) fail in this case, but slight modifications to some of the proofs overcome these difficulties. It is also possible to solve infinite systems of semilinear equations using these methods. Once again, some slight modifications to some of the hypotheses are needed, and we leave these to the interested reader. It is an elementary exercise to reformulate all of these results to solve equations in which the local nonlinearities $f_{k}$ are replaced with nonlocal nonlinearities of the form $f_{k}\left(D_{1} u_{1}(x), \ldots, D_{n} u_{n}(x)\right)$, where $f_{k}: \mathbf{R}^{n} \rightarrow \mathbf{R}$ and where $D_{k}=D_{k}(x, \cdot)$ is a positive kernel.

Appendix. At one crucial point in the proof of (2.7), we need the following fact. (All hypotheses and notations of $\S \S 1$ and 2 are assumed here.)

(A.1) Proposition. Let $z \in\{V J(b)>V \mu\}=\{a<0\} \subset\{J(a)=0\}$. If $V \mu(z)=V J(a)(z)$, then $V \mu(w)<V J(a)(w)$ for some $w$ in $F$.

This proposition depends on hypothesis (2.6), among others. There is undoubtedly a purely potential theoretic way to prove this result, but we resort to a proof using Markov processes. In order to do this, we apply some work of Bouleau [3]. 
Let $h=U \Psi$ : this function is finite on $F$ by (1.13) and is bounded away from zero on compacts in $F$ by (1.6i). Define two new kernels $Y$ and $C$ on $(F, \mathcal{F})$ by setting $Y(z, \cdot)=h^{-1}(z) U(z, \cdot)$ and $C(z, \cdot)=B(z, \cdot) h(\cdot)$. Recall that $Y$ is the zero potential $Y^{0}$ of a sub-Markovian resolvent $\left(Y^{\alpha}\right)$ on $(F, \mathcal{F})$. Hypothesis (1.0) implies that $\left(Y^{\alpha}\right)$ is the resolvent of a right Markov process on $(F, \mathcal{F})$. For more information about $\left(Y^{\alpha}\right)$, see [6]. For information about right processes, see [5 and 2]. Now let $\partial$ be a new point not contained in $F$, set $F^{\prime}=F \cup\{\partial\}$, and $\mathcal{F}^{\prime}=\sigma(\mathcal{F}, \partial)$. Extend the resolvent $\left(Y^{\alpha}\right)$ to $\mathcal{F}^{\prime}$ by setting $\alpha Y^{\alpha}(z,\{\partial\})=1-\alpha Y^{\alpha}(z, F)$ for every $z$ in $F$ and $\alpha Y^{\alpha}(\partial,\{\partial\})=1:\left(Y^{\alpha}\right)$ so extended is a Markovian resolvent on $\left(F^{\prime}, \bar{F}^{\prime}\right)$. Extend $C$ to $F^{\prime}$ by setting $C(z,\{\partial\})=0$ for every $z$ in $F$ and $C\left(\partial, F^{\prime}\right)=0$. Note that if we define

$$
Z^{\alpha}=\sum_{k=0}^{\infty}\left(Y^{\alpha} C\right)^{k} Y^{\alpha}
$$

then $Z f=h^{-1} V f$ for every $f \in \mathrm{p} \mathcal{F}^{\prime}$ with $f(\partial)=0$. Let us compute the function

$$
b=\sum_{k=0}^{\infty}(Y C)^{k} 1_{F^{\prime}}=1_{F^{\prime}}+h^{-1} \sum_{k=1}^{\infty}(U B)^{k} U \Psi \leq 1_{F^{\prime}}+h^{-1} V \Psi<\infty .
$$

The function $b$ is invariant for the resolvent $\left(Z^{\alpha}\right)[\mathbf{3}]$. Bouleau $[\mathbf{3}]$ shows that since $b$ is finite, the resolvent $\left(D^{\alpha}\right)$ defined by $D^{\alpha}(z, \cdot)=b^{-1}(z) Z^{\alpha}(z, \cdot) b(\cdot)$ is the resolvent of a right Markov process $X=\left(\Omega, G, G_{t}, X_{t}, \theta_{t},\left(P^{x}\right)_{x \in F^{\prime}}\right)$. We use this process to prove (A.1). Note that $D f=b^{-1} Z(b f)=b^{-1} h^{-1} V(b f)$, so $G=\{V J(b)>V \mu\}=$ $\left\{D\left(b^{-1} J(b)\right)>D\left(b^{-1} \mu\right)\right\}$ : this set is finely open for $X$. What does this mean? It means that if we define $T=\inf \left\{t>0: X_{t} \in G^{c}\right\}$, then $T>0 P^{z}$ almost surely whenever $z \in G$. Recall that $\mu=r \cdot d M+\mu^{s}$ and $r>0$ on $F$. By the strong Markov property,

$$
E^{z}\left[D b^{-1} r\left(X_{T}\right)\right]=E^{z} \int_{T}^{\infty} b^{-1} r\left(X_{s}\right) d s<E^{z} \int_{0}^{\infty} b^{-1} r\left(X_{s}\right) d s=D b^{-1} r(z),
$$

since $b^{-1} r>0$ and $T>0 P^{z}$ almost surely. Thus $E^{z}\left[D b^{-1} \mu\left(X_{T}\right)\right]<D b^{-1} \mu(z)=$ $D\left(b^{-1} J(a)\right)(z)$. Now compute

$$
\begin{aligned}
E^{z}\left[D\left(b^{-1} J(a)\right)\left(X_{T}\right)\right] & =E^{z} \int_{T}^{\infty} b^{-1} J(a)\left(X_{s}\right) d s=E^{z} \int_{0}^{\infty} b^{-1} J(a)\left(X_{s}\right) d s \\
& =D\left(b^{-1} J(a)\right)(z),
\end{aligned}
$$

since $J(a)\left(X_{s}\right)$ is zero provided $s<T$ (recall $J(a)=0$ on $G$ ). Thus we see

$$
E^{z}\left[D b^{-1} \mu\left(X_{T}\right)\right]<E^{z}\left[D b^{-1} J(a)\left(X_{T}\right)\right]
$$

and so $D b^{-1} \mu(w)<D b^{-1} J(a)(w)$ for some $w$ in $F$. That is, $V \mu(w)<V J(a)(w)$. Q.E.D.

\section{REFERENCES}

1. P. Baras and M. Pierre, Singularités eliminables pour des équations semi-linéaires, Ann. Inst. Fourier 34 (1984), 185-206.

2. R. M. Blumenthal and R. K. Getoor, Markov processes and potential theory, Academic Press, New York, 1968.

3. N. Bouleau, Théorie du potentiel associée à certains systèmes différentiels, Math. Ann. 255 (1981), $335-350$. 
4. H. Brezis, Problèmes elliptiques et paraboliques non linéaires avec données mesures, Séminaire Goulaouic-Meyer-Schwartz 1981-1982, XX.1-XX.12.

5. R. K. Getoor, Markov processes: Ray processes and right processes, Lecture Notes in Math., vol. 440, Springer-Verlag, Berlin-Heidelberg-New York, 1975.

6. R. K. Getoor and Joseph Glover, Markov processes with identical excessive measures, Math. Z. 184 (1983), 287-300.

7. Joseph Glover and P. J. McKenna, Solving semilinear partial differential equations with probabilistic potential theory, Trans. Amer. Math. Soc. 290 (1985), 665-681.

8. P. L. Lions, On the existence of positive solutions of semilinear elliptic equations, SIAM Review 24 (1982), 441-467.

9. P. A. Meyer, Probability and potentials, Blaisdell, Waltham, Mass., 1966.

10. C. V. Pao, On nonlinear reaction-diffusion systems, J. Math. Anal. Appl. 87 (1982), 165-198.

11. W. Walter, Differential and integral inequalities, Ergeb. Math. Grenzgeb., vol. 55, SpringerVerlag, Berlin and New York, 1970.

Department of MAThematics, University of Florida, Gainesville, Florida 32611 